\title{
El proceso de decisión compartida en el tratamiento del paciente psiquiátrico: estudios empíricos y evaluación de la capacidad.
}

"The shared decision-making process in the treatment of psychiatric patient: empirical studies and capacity assessment".

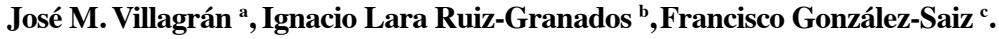 \\ a, by c Psiquiatra. Unidad de Gestión Clínica de Salud Mental. Hospital de Jerez de la Frontera, Cádiz. Ser- \\ vicio Andaluz de Salud, España. ${ }^{a}$ Coordinador de la Unidad de Hospitalización Psiquiátrica. ${ }^{b y c}$ Unidad \\ de Salud Mental Comunitaria Sierra de Cádiz.
}

Correspondencia: José M. Villagrán (jmaria.villagran.sspa@juntadeandalucia.es)

Recibido: 28/08/2013; aceptado: 09/02/2014

\begin{abstract}
RESUMEN: Existe investigación empírica sobre experiencias de decisión compartida en salud mental, preferentemente en depresión y esquizofrenia, y algo menos en adicciones. La evidencia disponible a partir de los estudios llevados a cabo en los últimos años muestra que esta práctica mejora la calidad de las decisiones y parece efectiva en el proceso decisorio (aumenta el conocimiento y la participación, favorece una mayor congruencia con los valores y preferencias del paciente), aumentando, generalmente, la satisfacción del usuario. Sin embargo, existe escasa o mínima evidencia acerca de su eficacia sobre indicadores de resultados de salud. Uno de los elementos claves de los procesos de decisión compartida es la determinación de la capacidad del paciente para tomar la decisión clínica en cuestión. Se trata de un juicio clínico que posee un papel relevante en el difícil equilibrio entre autonomía y beneficencia/no maleficencia en la atención sanitaria. Así, una adecuada evaluación de la capacidad de tomar decisiones del paciente debe servir para proteger ambos principios y evitar dos tipos de errores: por un lado, el soslayar injustificadamente la autonomía del paciente para salvaguardar su bienestar; por otro, el injustificable respeto a su autonomía al precio de su bienestar.
\end{abstract}

PALABRAS CLAVE: toma de decisiones, psiquiatría, tratamiento, ética, capacidad.
ABSTRACT: There is empirical research on shared decision making in mental health, above all in depression and schizophrenia, and somewhat less in addictions. Studies carried out in recent years produce evidence showing that it improves the quality of decisions and it seems to be effective in decision making (increased knowledge and participation, promotes greater consistency with the patient values and preferences), increasing user satisfaction mostly. However, there is tiny evidence of its effectiveness on health outcomes. One of the key elements of shared decision-making processes is the determination of the patient's capacity to make the clinical decisions in question. This is an assessment that has a core role in the delicate balance between autonomy and beneficence / non-maleficence in health care. Thus, a proper assessment of the capacity of the patient to make decisions might be useful to protect both principles and to avoid two types of errors: first, to ignore unjustifiably the patient autonomy to safeguard their welfare, and secondly, the unwarranted respect for their autonomy for the price of their welfare.

KEY WORDS: decision making, psychiatry, therapeutics, medication adherence, ethics, mental competency. 
1. Estudios empíricos sobre la aplicación del proceso de decisión compartida $(P D C)$ en salud mental.

La práctica clínica suele implicar la instauración de un tratamiento individualizado tras un adecuado proceso diagnóstico. El momento previo a la intervención terapéutica supone la adopción de una o más decisiones en la que el paciente debe implicarse de forma activa. Se ha apuntado que los procesos de decisión comparti$d a(\mathrm{PDC})$ en la atención sanitaria podrían constituir un nuevo y prometedor marco para configurar la relación médico-paciente del siglo XXI, una vez asumidas las críticas a la organización de la atención sanitaria actual y la subsiguiente desconfianza de los ciudadanos con la profesión médica. En los últimos años se han publicado exhaustivas revisiones sobre investigación sobre PDC y salud mental (1-2), especialmente en pacientes con trastornos depresivos y trastornos mentales graves (preferentemente, esquizofrénicos) y, en menor medida, en drogodependientes. A continuación se analizan los trabajos más relevantes.

\subsection{Depresión}

Von Korff et al (3) compararon, en un ensayo aleatorizado, dos estrategias de intervención en pacientes depresivos atendidos en atención primaria que respondían a tratamiento inicial pero con alto riesgo de recaída. El programa de prevención de recaídas, de 12 meses de duración y que incluía, entre otros elementos, múltiples sesiones de PDC, produjo un mayor efecto, aunque de magnitud moderada, sobre los síntomas depresivos y, consecuentemente, sobre algunas medidas de discapacidad, pero no arrojó diferencias en tasas de recaídas al año con el grupo control de cuidados rutinarios.

Loh et al (4), por su parte, realizaron un ensayo aleatorizado por clusters para estudiar el efecto de una intervención con PDC en más de 400 pacientes depresivos. Al cabo de las 6-8 semanas de seguimiento, el grupo de la intervención mostró una mayor participación en la toma de decisiones y una mayor satisfacción con la atención recibida que el grupo control. Sin embargo, no se encontraron diferencias en cuanto a la gravedad de los síntomas depresivos.

\subsection{Adicciones}

Joosten et al, (5) llevaron a cabo un ensayo aleatorizado por clusters en el que estudiaron los efectos de una intervención que incluía PDC en 220 pacientes hospitalizados con dependencia a sustancias psicoactivas. Durante los 3 meses de seguimiento, el grupo de pacientes que recibió la intervención mostró una disminución significativa del consumo de sustancias y de la severidad de los síntomas psiquiátricos. 
ORIGINALES Y REVISIONES

\subsection{Esquizofrenia.}

A mediados de los 80, Bradford et al (6) publican un estudio en el que analizan el proceso de decisión en pacientes con trastornos mentales graves (preferentemente trastornos del ánimo, si bien ninguno diagnosticado de esquizofrenia). Concluyeron que una mayor severidad del trastorno se asociaba con mayor conflicto decisorio, menor nivel de confianza en la decisión, mayor nivel de irracionalidad en la decisión y mayor alteración del proceso de información.

Bunn et al (7) publican un estudio en el que exploran el PDC en una muestra de 94 pacientes esquizofrénicos ambulatorios estabilizados, focalizado en la opción de continuar o no con el tratamiento antipsicótico depot. Previamente a la toma de decisión, se les proporcionó a los pacientes información sobre pros y contras de continuar o discontinuar esta pauta. El 87\% (82 pacientes) de la muestra decidió continuar en la terapia, mientras que el 10\% (9 pacientes) se mostró indeciso. Únicamente 3 pacientes decidieron dejar el tratamiento depot. Se compararon ambos grupos respecto del conflicto en la toma de decisión, la autoeficacia percibida sobre la misma, el apoyo emocional para tomarla y la percepción de los riesgos y beneficios, encontrándose diferencias significativas entre ambos grupos en el sentido de un mayor conflicto decisorio y una menor autoeficacia y apoyo emocional en la decisión, además de menores expectativas de hospitalización si se discontinuaba el tratamiento y menos expectativas de beneficios y mayores de efectos secundarios si continuaban con el tratamiento en los indecisos. A pesar de sus limitaciones (no existencia de grupo control, alta proporción de pacientes continuadores, posiblemente influida por el tipo de paciente), este estudio canadiense fue el primero en evaluar el PDC en pacientes esquizofrénicos.

Malm et al (8), mediante un ensayo clínico aleatorizado con 84 pacientes esquizofrénicos ambulatorios, compararon dos programas terapéuticos basados en la comunidad. Al cabo de dos años, el programa que incluía entre sus intervenciones el PDC mejoraba significativamente la función social y producía una mayor satisfacción del usuario.

Van Os et al (9) estudiaron la utilización de una intervención aislada, dirigida a pacientes esquizofrénicos y sus psiquiatras, que favorecía la decisión compartida (2-COM, listado de 20 necesidades comunes). Se aleatorizaron 134 pacientes esquizofrénicos entre un grupo que recibía el cuidado habitual y un grupo con esta intervención. Los que se asignaron a ésta, expresaron una mejoría estable en la comunicación médico-paciente y cambios inmediatos en el manejo de la medicación, en comparación con el grupo control.

Hamann et al (10) publicaron el primer ensayo clínico aleatorizado sobre la aplicación del PDC en pacientes esquizofrénicos hospitalizados. Se comparó la aplicación de este modelo con el cuidado habitual en una muestra de 107 pacientes esquizofrénicos en fase aguda que habían sido hospitalizados en 12 unidades 
de hospitales estatales de Munich, Alemania. A todos los pacientes asignados el grupo PDC se les pudo aplicar el modelo. Los pacientes asignados a este grupo obtuvieron un mejor conocimiento de su enfermedad y una mayor implicación en la toma de decisiones que los del grupo control, aunque este último efecto fue disminuyendo con el tiempo. Así mismo, se mejoró la actitud hacia el tratamiento y se favoreció la participación en actividades de psicoeducación e intervenciones socioterapéuticas. El seguimiento de estos pacientes a los 6 y 18 meses tras el alta no arrojó diferencias entre los grupos en cuanto a cumplimiento con el tratamiento (en ambos fue pobre), aunque sí una tendencia favorable al grupo PDC en cuanto a tasa de rehospitalización. Curiosamente, aquellos pacientes con mayores expectativas de participación y mayor deseo de autonomía reingresaron más frecuentemente. Una posible explicación de los datos es que la intervención, administrada en una sola ocasión, "tan pronto como fue posible", tuvo un efecto escasamente duradero y poco determinante en un tratamiento a largo plazo, mientras que una intervención con varias sesiones de refuerzo y una orientación especialmente a esos pacientes con altas expectativas de participación, podrían haber mejorado los resultados (11). En un ensayo aleatorizado por clusters, Priebe et al (12) estudiaron el uso de una intervención a través de ordenador (DIALOG) para estructurar las interacciones médico-paciente con respecto a la calidad de vida y las necesidades de cuidados cada 2 meses durante un año. Se aleatorizaron 134 clínicos de 6 países entre esta intervención y el tratamiento habitual y se incluyeron más de 500 pacientes esquizofrénicos o con trastornos relacionados. Los pacientes adscritos al grupo experimental expresaron una mayor calidad de vida subjetiva, un menor número de necesidades no cubiertas y una mayor satisfacción con el tratamiento en un año que los del grupo control.

Recientemente, Hamann et al (13) realizaron un estudio en el que 61 pacientes esquizofrénicos ingresados en agudos fueron aleatorizados entre recibir entrenamiento en PDC o entrenamiento cognitivo. Los primeros mostraron mayor preferencia por participar y mayor deseo de responsabilizarse en las decisiones terapéuticas, lo que se mantuvo a los 6 meses. Por contra, se mostraron más escépticos ante el tratamiento y fueron percibidos por sus psiquiatras como "más problemáticos".

Finalmente, Woltmann et al (14) condujeron un diseño aleatorizado por clusters en el que evaluaron el uso de un sistema electrónico de apoyo a la decisión en tres unidades de salud mental comunitaria. Un total de 20 gestores de casos fueron asignados aleatoriamente entre el grupo de intervención y un grupo control con tratamiento habitual. Ochenta pacientes gestionados por ellos, en su mayoría esquizofrénicos o con trastornos afectivos graves, fueron asignados a los mismos grupos que sus gestores. Los gestores del grupo experimental se mostraron significativamente más satisfechos con el proceso de planificación de cuidados, mientras que 
ORIGINALES Y REVISIONES

los usuarios de este grupo mostraron un mayor recuerdo del plan de cuidados tres días después de la sesión de planificación que los del grupo control. Sin embargo, entre los usuarios de uno y otro grupo no hubo diferencias significativas en cuanto a la satisfacción.

Como conclusión, la evidencia disponible a partir de los estudios empíricos llevados a cabo en los últimos años, muestra que la aplicación de PDC en salud mental mejora la calidad de las decisiones y parece efectivo en el proceso decisorio. Específicamente aumenta el conocimiento y la participación, observándose una mayor congruencia con los valores y preferencias del paciente, así como una cierta satisfacción del usuario. Sin embargo, existe escasa o mínima evidencia acerca de su eficacia en medidas de resultados de salud, precisándose estudios a largo plazo que exploren esta relación (15).

\section{La cuestión de la capacidad en la decisión compartida}

Uno de los elementos claves de los procesos de consentimiento informado y decisión compartida es la determinación de la capacidad del paciente para tomar la decisión clínica en cuestión. Se trata de un juicio que el clínico lleva a cabo a partir de datos de entrevistas exploratorias y que, como todo juicio clínico, tiene carácter inferencial, probabilístico. Este juicio del clínico posee un papel relevante en el difícil equilibrio entre autonomía y beneficencia/no maleficencia en la atención sanitaria. Así, una adecuada evaluación de la capacidad de tomar decisiones del paciente debe servir para proteger ambos principios y evitar dos tipos de errores: por un lado, el soslayar injustificadamente la autonomía del paciente para salvaguardar su bienestar; por otro, el injustificable respeto a su autonomía al precio de su bienestar (16).

Es preciso distinguir entre capacidad legal o de derecho (competency en inglés) y capacidad natural o de hecho (capacity). La primera es un concepto jurídico que se refiere al reconocimiento legal de las aptitudes psicológicas de alguien para tomar una decisión y, por tanto, para desempeñar su autonomía moral. La segunda es un concepto psicológico que se refiere a dichas aptitudes psicológicas para tomar aquí y ahora la decisión. Calificamos de incapaz (de hecho) a aquella persona cuyas aptitudes psicológicas para tomar una decisión están alteradas aquí $\mathrm{y}$ ahora (y, por tanto, pierde, aunque sea transitoriamente, su capacidad de derecho) y de incapacitado (de derecho) a aquella persona cuya capacidad de derecho está abolida permanentemente (aunque no necesariamente de forma irreversible, ver infra) a partir de una sentencia judicial de incapacitación debida a la existencia de alguna alteración mental o cerebral que altera de forma habitual y no meramente 
circunstancial o episódica su capacidad de hecho (estados vegetativos permanentes, psicosis con gran deterioro cognitivo, demencias avanzadas) (17).

Por principio, a toda persona mayor de edad en una situación clínica se le supone capacidad de hecho (y, por tanto, es susceptible de tomar parte en un proceso de decisión compartida) salvo que se demuestre lo contrario o exista previamente una sentencia de incapacitación. Por tanto, es la incapacidad lo que hay que demostrar y esto también es aplicable para los pacientes psiquiátricos, incluidos los afectados de trastornos mentales graves. La idea de que estos pacientes precisan una actitud paternalista y no son capaces de tomar decisiones clínicas no está justificada (18).

Incluso en los casos de pacientes psiquiátricos incapacitados de derecho, es preciso determinar el grado de capacidad en un contexto dado para una decisión particular. A este respecto, la propia definición de capacidad legal, de derecho o de iure ha pasado, en los últimos años, de considerar la capacidad como una condición global, aplicable a cualquier contexto, a considerarla una condición más específica, restringida a contextos particulares de toma de decisiones (16). Así, mientras que un paciente capacitado de iure puede ser incapaz, en un momento dado, para tomar determinadas decisiones, lo contrario también podría darse (aunque sea menos frecuente), un incapacitado de iure podría ser capaz de tomar ciertas decisiones clínicas.

Otra consecuencia de esta concepción de capacidad más restringida es que las categorías diagnósticas no pueden resolver la cuestión de la capacidad para tomar decisiones clínicas, ya que las habilidades funcionales que son relevantes para ésta (comprensión, apreciación, razonamiento, expresión de la elección) son lógicamente independientes de la mayor parte de aquéllas (18-19). En este sentido, la capacidad para tomar decisiones clínicas, entendida como la aptitud psicológica para evaluar la información recibida y comprender su relevancia y significado en el contexto del paciente, no es un fenómeno de todo o nada, sino un continuum que puede variar con cada paciente e, incluso, en distintos momentos en el mismo paciente (20).

Ganzini et al (21-22) analizaron los errores más frecuentes entre profesionales a la hora de la evaluación de la capacidad para tomar decisiones sobre el tratamiento y apuntaron como el error más frecuente (y que precisaría una mayor atención pedagógica) la creencia de que si una persona muestra incapacidad para tomar un determinado tipo de decisión clínica, también será incapaz en todas las demás. Abundando en esto, señalan 10 mitos con respecto a la capacidad de tomar decisiones, que quedan recogidos en la Tabla 1.

La evaluación de la capacidad para tomar decisiones de tratamiento en la situación clínica es una cuestión aún no resuelta (17). Aunque los médicos han realizado evaluaciones de la capacidad de los pacientes desde siempre, hasta la promulgación de la Ley 41/2002 en España (en especial sus artículos 5.3 y 9.3.a), no tenían soporte legal, lo que paradójicamente poseían los notarios en el Código 
ORIGINALES Y REVISIONES

Civil desde hace lustros. La Ley 41/2002 otorga exclusivamente al médico responsable del paciente la responsabilidad de esta evaluación. Nótese que psicólogos o psiquiatras únicamente tendrían una función pericial en caso de que el médico responsable precisara de ayuda (17).

Tabla 1 .

Diez mitos sobre la capacidad para tomar decisiones (22).

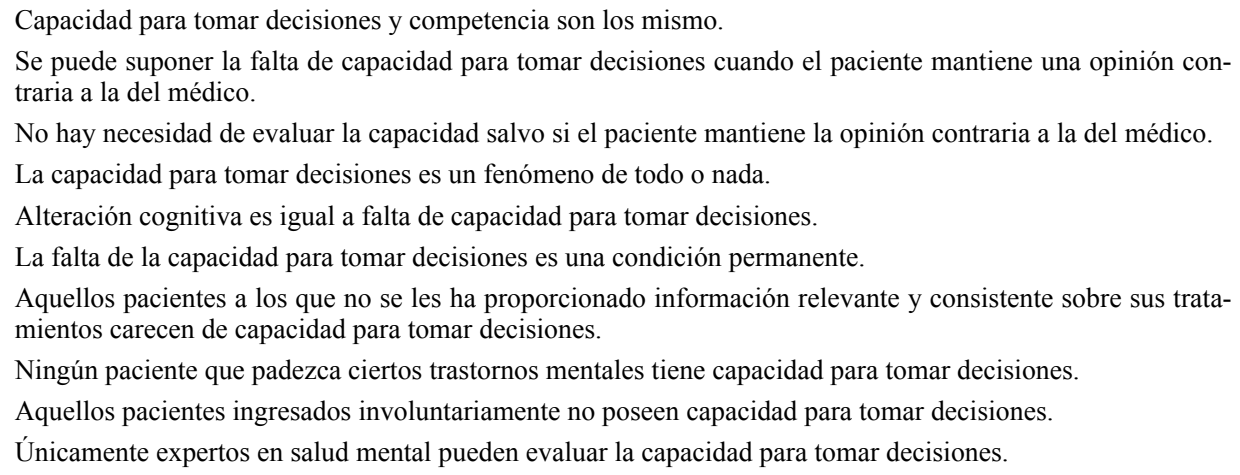

La evaluación de la capacidad para tomar decisiones del paciente debe llevarse a cabo siempre que concurran una serie de circunstancias: cambio brusco de estado mental (ya sea por alteraciones psiquiátricas o médicas, metabólicas, neurológicas, intoxicaciones, etc); rechazo a tratamiento indicado escasamente argumentado; aceptación de tratamientos invasivos sin ponderación de riesgos o existencia de trastorno mental o neurológico conocido que pudiera alterar transitoriamente la capacidad de hecho. A esto se añade alguna otra situación específica como la elaboración o ejecución de directrices anticipadas, la participación en investigación clínica o, en los países en donde es legal, el suicidio asistido (16).

\subsection{Criterios o estándares para la evaluación de la capacidad para tomar decisiones \\ Tradicionalmente, en nuestro medio, la evaluación de la capacidad se ha rea- lizado de facto mediante la aplicación del sentido común y una sucinta exploración cognitiva o psicopatológica. No han existido procedimientos estandarizados o he- rramientas desarrolladas para tal fin. Sin embargo, en otros países, como EEUU, ya desde finales de los 70 se establecieron una serie de criterios que, apenas modifica- dos, han conformado los 4 estándares ampliamente aceptados para la evaluación de la capacidad para tomar decisiones de tratamiento, al menos desde su perspectiva psiquiátrica, $\mathrm{y}$, además, la necesidad de tener en cuenta para ello el tipo de decisión}


en cuestión (23). Los estándares surgidos, en un primer momento, para la evaluación de la capacidad en el consentimiento para participar en investigación clínica (24) son: la capacidad para realizar y expresar una elección, la capacidad para comprender la información relevante, la capacidad para apreciar correctamente la situación y las posibles consecuencias de su decisión, y la capacidad para manejar racionalmente la información y las distintas opciones (25).

A finales de los 80 , desde la neurología, se señala que, además de las funciones psicológicas mencionadas, sería preciso evaluar el funcionamiento de los sustratos neurobiológicos subyacentes y apuntan una serie de áreas (atención, memoria, lenguaje, funciones ejecutivas, afectividad) que deberían ser evaluadas (26-27).

Finalmente, distintos autores han abundado en la idea original de Roth et al (23) de que la capacidad se relaciona directamente con el tipo concreto de decisión que hay que tomar. Así, en decisiones clínicas complejas en donde la relación riesgo/beneficio no sea fácil de establecer, se precisará un nivel de capacidad más alto que en situaciones clínicas simples, donde el nivel de capacidad requerido podría ser más bajo. Es lo que Drane denominó escala móvil de capacidad (28-29), señalando que la determinación de la capacidad es asimétrica y, por tanto, no existe un punto de corte fijo sino que dependerá del nivel de dificultad de la decisión. Así, en decisiones de nivel I (baja dificultad), la mayoría de las personas serían consideradas capaces, mientras que en las de nivel III (alta complejidad), muchas serían incapaces.

A pesar del indudable éxito de la propuesta de la escala móvil, existe un interesante debate sobre si la valoración de la complejidad de la situación dependiente de los clínicos es o no una forma subrepticia de reintroducir el paternalismo en la toma de decisiones $(17,30)$.

\subsection{Instrumentos de evaluación de la capacidad}

Aunque el juicio clínico sigue siendo el "patrón oro" en la evaluación de la capacidad de un paciente para tomar decisiones relacionadas con el tratamiento (31), también sigue siendo idiosincrásico y, muchas veces, poco fiable (32), en lo que abunda la inexistencia de directrices internacionalmente aceptadas (33). Es por ello por lo que la utilización de instrumentos de evaluación de dicha capacidad como ayuda al juicio del clínico puede ser de suma importancia.

Existen exhaustivas revisiones sobre las herramientas utilizadas en la evaluación de la capacidad, tanto en contextos investigadores como clínicos (31,34-35). Los instrumentos usados en la evaluación de la capacidad han sido de 3 tipos (17): tests neuropsicológicos (preferentemente el MMSE - Mini Mental State Examination (36), los protocolos específicos de evaluación de la capacidad (dirigidos a la evaluación en un contexto determinado, por ejemplo, en la terapia electroconvulsiva, CIS - Competence Interview Schedule, (37) o en la elaboración de directrices 
ORIGINALES Y REVISIONES

anticipadas HCAT - Hopkins Competency Assessement Tool), (38) y los protocolos generales de evaluación (que incluyen los listados de preguntas o guiones de entrevistas, las viñetas estandarizadas y los protocolos propiamente dichos).

Los principales protocolos propiamente dichos son: $C C T I$ - Capacity to Consent to Treatment Instrument (32), CAT - Capacity Assessment Tool (39), HCAI - Hopemont Capacity Asessment Interview (40), DAM - Decision Assessment Measure (41), $A C E$ - Aid to Capacity Evaluation (42), y, especialmente, MacCATMacArthur Competence Assessment Tool (43).

Aunque el protocolo MacCAT (del que existen tres versiones, una para investigación - MacCAT-CR, otra para ámbitos forenses - MacCAT-CA, y finalmente la versión para tratamiento - Mac CAT-T, con adaptación española en Hernando et al, (44)) es el que posee mayor sustento empírico, otros instrumentos podrían tener ventajas según qué contextos (34-35). Por ejemplo, en una situación de evaluación o screening inicial, podrían ser más aconsejables ACE o HCAI, no así en una evaluación global, continuada o más exhaustiva, en donde el MacCAT-T sería de elección. Ante pacientes con enfermedades con graves alteraciones cognitivas, además del MacCAT-T (más en población psiquiátrica) podrían ser más útiles CCTI (en demencias) o HCAI (en contextos asistenciales de larga estancia), y en el contexto de la terapia electroconvulsiva, como se vio, el CIS.

Todos los instrumentos tiene sus limitaciones $(17,31,34)$, En primer lugar, la cuestión de su validez: la mayoría de estos instrumentos únicamente han sido testados una sola vez y con muestras pequeñas. Además, el "patrón oro" utilizado como comparador de referencia es la opinión de un clínico experimentado, opinión que se sabe que puede ser idiosincrásica. En segundo lugar, las propiedades psicométricas de los instrumentos son muy variables y la concordancia entre ellos poco concluyente, siendo mayor para el criterio de comprensión que para el de apreciación. Por otro lado, los déficits en comprensión y razonamiento tienen una alta correlación con los rendimientos detectados por los tests neuropsicológicos, lo cual no ocurre para los déficits en la apreciación y la expresión de la elección.

\subsection{Evaluación de la capacidad en psiquiatría.}

La cuestión de la capacidad para tomar decisiones sobre el tratamiento se aplica, como hemos visto, entre otros, a los pacientes psiquiátricos. Estas consideraciones deben hacerse al tiempo que se aplica el marco legal que rige los ingresos y los tratamientos involuntarios (45). En Reino Unido, por ejemplo, coexisten la Mental Health Act de 1983 y la Mental Capacity Act de 2005, ambas enmendadas por la Mental Health Act de 2007. La primera establece un marco legal para el ingreso y el tratamiento involuntarios si existe una enfermedad mental y un riesgo para sí o para otros. La segunda establece un marco legal más amplio en el que se regula la toma de decisiones (incluidas las de tratamiento) en aquellas personas que 
no poseen capacidad para decidir. Mientras que la primera se preocupa principalmente de reducir el riesgo para sí u otros (principio de beneficencia), la segunda está orientada a favorecer que la persona tome sus propias decisiones y que éstas reflejen sus deseos y su mejor interés, obteniendo la atención menos coercitiva posible (principio de autonomía) (46).

En España, de un modo similar pero con un grado de indefinición mucho mayor, los ingresos involuntarios están regulados por el artículo 763 de la Ley de Enjuiciamiento Civil 1/2000. Este artículo se incluye en el capítulo II de la ley, titulado "De los procesos sobre la capacidad de las personas", pero en el que únicamente se regulan los procedimientos judiciales para la declaración de incapaz de derecho. El artículo 763, sin embargo, versa sobre el "internamiento no voluntario por razones de trastorno psíquico", y en él, nuevamente, se regulan los procedimientos para realizar un internamiento involuntario pero sin especificar los criterios que deben aplicarse por parte del facultativo, salvo que la persona "no esté en condiciones de decidirlo por sí". Por su parte, la cuestión de la capacidad se incluye en La Ley 41/2002 Básica Reguladora de la Autonomía del Paciente, en su artículo 9 relativo a los límites del consentimiento informado, incluyendo la falta de capacidad de hecho y de derecho como supuestos en los que se puede aplicar el consentimiento por representación.

La existencia de dos marcos legales de filosofías contrapuestas que intentan proveer de estructuras de decisión sustitutivas en relación a la atención sanitaria y el tratamiento genera dilemas éticos, pero también problemas prácticos (46). El hecho de que exista una legislación específica para ingresar y tratar contra su voluntad a los enfermos de patología mental (y no a los de patología física) es ya de por sí una fuente de discriminación y está insuficientemente justificada desde el punto de vista ético.

Por otro lado, cuatro situaciones posibles podrían darse ante un eventual ingreso psiquiátrico: a) que el paciente sea capaz de hecho para tomar decisiones sobre su ingreso pero que, a pesar de ello, se le ingrese involuntariamente por aplicación del artículo 763 de la LEC; b) que el paciente sea incapaz de hecho pero que, a pesar de ello, se le ingrese de forma voluntaria; c) que el paciente sea capaz y voluntariamente acepte el ingreso; y d) que el paciente sea incapaz y se le ingrese involuntariamente. La frecuencia de cada tipo puede variar según los contextos sanitarios, pero algunos estudios $(45,47)$ apuntan a que las situaciones más frecuentes son la "c" y la "d" (aproximadamente un tercio de los ingresos cada una). El otro tercio se repartiría entre las opciones "b" (entre un 24-39\%) y la "a" (entre el 4-10\%) (45, 47-48). Mientras que la situación "c" no generaría problemas, las otras son problemáticas, especialmente la "a" y la "b".

El porcentaje de ingresos psiquiátricos en unidades de agudos en los que el paciente es incapaz (opciones "b" y "d" de las anteriores) y evaluados específi- 
ORIGINALES Y REVISIONES

camente mediante el MacCAT-T, suele oscilar según los estudios, entre el 29 y el $60 \%(47,49-50)$, lo que supone un porcentaje algo superior que en los ingresos agudos de otras especialidades (entre el $25-40 \%)(49,51)$. Generalmente las patologías que más contribuyen a este tipo de ingresos de incapaces son la manía y la esquizofrenia, siendo mucho menos frecuentes las depresiones y los trastornos de la personalidad (47). Los datos existentes apuntan a que únicamente uno de cada 4 pacientes incapaces son detectados por los facultativos al ingreso (49).

Ante una situación como la "b", un paciente incapaz de hecho pero al que se le ingresa de forma voluntaria, la mayor parte de las veces los clínicos se sitúan en una tierra de nadie: por un lado, en virtud de la Ley de Autonomía del paciente, habría que cuidar el que las decisiones que éste tome sean respetadas y se actúe en el mejor beneficio del paciente y de la forma menos coercitiva, pero por otro, en el caso que el clínico sospeche de la incapacidad del paciente para tomar decisiones como el rechazo al tratamiento, la no aplicación del artículo 763 y de sus garantías legales, puede llevar a la aplicación de prácticas meramente paternalistas y coercitivas. Los datos de los estudios empíricos reflejan esta percepción. Por ejemplo, en el estudio de Owen et al (45), los pacientes incapaces ingresados voluntariamente mostraron un mayor nivel de rechazo al tratamiento, coerción percibida, presiones para el tratamiento y una menor sensación de respeto por su opinión que los capaces ingresados voluntariamente.

La situación "a" (paciente capaz para tomar decisiones pero ingresado involuntariamente) plantea un problema diferente. Aquí estamos ante la privación de libertad de una persona que padece un trastorno psíquico (esta situación no se puede dar ante una enfermedad física) que no tiene alterada su capacidad para decidir si quiere estar o no ingresado, si quiere o no tratamiento. En puridad, se trata de una contradicción en términos, ya que el propio artículo 763 de la LEC exige, ante el ingreso involuntario, además de la existencia del trastorno psíquico, que la persona "no esté en condiciones de decidirlo por sí", lo que podría suponer la incapacidad. La interpretación alternativa, la más frecuente, es aplicar el 763 por otras razones distintas de la capacidad o incapacidad decisoria del paciente psiquiátrico, en particular, la protección social (riesgo de daño del paciente a sí o a otros). Aunque la ley española no lo explícita, sí lo hacen las leyes británicas y de otros países (46). Esto tiene importantes consecuencias. Por un lado, una definición laxa de trastorno psíquico podría hacer que numerosas pacientes capaces pudieran ser ingresados contra su voluntad mediante el artículo 763 (esto está ocurriendo con los denominados trastornos de personalidad -límites, disociales, en donde prima la consideración de orden social sobre la falta de capacidad) (52). Pero, además, únicamente a las personas capaces que padezcan un trastorno psíquico se les puede ingresar y tratar contra su voluntad, lo que introduce una patente discriminación con los enfermos físicos. 
La propia situación "d", paciente psiquiátrico incapaz e ingresado involuntariamente, aunque legal, implica automáticamente la aplicación del artículo 763 (o de la Mental Health Act en Inglaterra y Gales), sin que el clínico tenga que atender a las garantías de consentimiento por representación incluidas en la Ley 41/2002 Básica de Autonomía del Paciente, adoptando la decisiones de tratamiento y de ingreso de forma unilateral y muchas veces francamente coercitiva, una vez autorizado el ingreso por la instancia judicial correspondiente. Aquí, como en el caso anterior, predomina el objetivo de protección social sobre la consideración de la capacidad decisoria del paciente, para justificar la aplicación de la atención involuntaria.

¿Existe alguna forma de superar esta situación? Algunos autores han propuesto la existencia de un solo marco unitario basado en la incapacidad de tomar decisiones (53). Así, la inevitable batalla entre autonomía y paternalismo tendría lugar en igualdad de condiciones, fuera cual fuese la fuente de incapacidad (esquizofrenia, demencia, intoxicación, coma) o la naturaleza del trastorno subyacente (mental o físico) (46). Sin embargo, esta propuesta no está exenta de dificultades.

En primer lugar, se necesitaría una sólida definición de incapacidad, aplicable a todo tipo de trastornos, incluidos los psiquiátricos, y una evaluación de la misma ágil y factible en el contexto sanitario. La legislación sobre ingresos involuntarios está diseñada, fundamentalmente, para favorecer el tratamiento e ingresos urgentes, a la luz del objetivo principal de inmediata protección social. Por el contrario, el marco garantista del consentimiento por representación en incapaces tiene un tempo diferente, y el establecimiento de las salvaguardas de la privación de libertad son más numerosas y costosas (54), por lo que un marco unitario debería ser lo suficientemente flexible como para satisfacer ambas necesidades. Pero la principal dificultad es la voluntad política de los gobiernos para ceder el instrumento de control social que supone el ingreso involuntario, ya que, bajo el pretexto de la protección social y la alusión al "trastorno psíquico", disponen de un elemento de detención preventiva que soslaya la exigencia de haber cometido un delito incluida en la Convención Europea de Derechos Humanos, aspectos que se diluirían si se diera un marco unitario basado en la incapacidad y el principio de no discriminación. Lo ocurrido en Gran Bretaña ilustra claramente esta cuestión: las sugerencias del Comité de Expertos de Inglaterra y Gales a finales de los 90 para reformar la Mental Health Act de 1983, vigente hasta entonces, se realizaron bajo la perspectiva del marco unitario, sugiriendo que en una primera fase, la determinación de incapacidad fuera necesaria. Esto nunca fue aceptado por el gobierno Laborista de entonces que pretendía una modificación en el sentido de ampliar el tipo de trastorno a los que aplicar la posibilidad de tratamiento compulsivo $(46,55)$. 
ORIGINALES Y REVISIONES

\section{Resumen}

Los trabajos realizados hasta el momento sobre experiencias de decisión compartida en salud mental ponen de manifiesto una mejoría en aspectos como la calidad de las decisiones y la satisfacción de los usuarios, aunque son necesarios más trabajos para poder concluir que también mejoran los indicadores de resultados en salud. La determinación de la capacidad del paciente para tomar la decisión clínica resulta fundamental mantener el difícil pero necesario equilibrio entre autonomía y beneficencia/no maleficencia en la atención sanitaria.

\section{BIBLIOGRAFÍA}

(1) Simon D, Wills CE, Harter M. Shared decision-making in mental health. En: Edwards A, Elwyn G editors. Shared decision-making in health care: Achieving evidence-based patient choice. 2nd ed. Oxford: Oxford University Press, 2009; p. 269-276.

(2) Duncan E, Best C, Hagen S. Shared decision making interventions for people with mental health conditions. Cochrane Database Syst Rev 2010, Issue 1. Art. No.: CD007297. DOI: 10.1002/14651858.CD007297.pub2.

(3) Von Korff M, Katon W, Rutter C, Ludman E, Simon G, Lin E, Bush T. Effect on disability outcomes of a depression relapse prevention program. Psychosomatic Medicine 2003; 65:938943.

(4) Loh A, Simon D, Wills CE, Kriston L, Niebling W, Härter M. The effects of a shared decision making intervention in primary care of depression: a cluster-randomized controlled trial. Pat Educ Couns 2007; 67:324-332.

(5) Joosten EA, De Jong CA, de Weert-van Oene GH, Sensky T, van der Staak CP. Shared decision-making reduces drug use and psychiatric severity in substance-dependent patients. Psychother Psychosom 2009; 78,4:245-253.

(6) Bradford MHB, Mann L, Kalucy RS. Psychiatric disturbance and decision-making. Aust N Z J Psychiatry 1986; 20:2210-217.

(7) Bunn MH, O'Connor AM, Tansey MS, Jones BD, Stinson LE. Characteristics of clients with schizophrenia who express certainty or uncertainty about continuing treatment with depot neuroleptic medication. Arch Psychiatr Nurs 1997; 11:238-248.

(8) Malm U, Ivarsson B, Allebeck P, Falloon IRH. Integrated care in schizophrenia: a 2-year randomized controlled study of two community-based treatment programs. Acta Psychiatr Scand 2003; 107:415-423.

(9) Van Os J, Altamura C, Bobes J, Gerlach J, Hellewell JSE, Kasper S, Naber D, Robert P. Evaluation of the Two-Way Communication Checklist as a clinical intervention. Results of a multinational, randomised controlled trial. Br J Psychiatry 2004, 184:79-83.

(10) Hamann J, Langer B, Winkler V, Busch R, Cohen R, Leucht S, Kissling W. Shared decision-making for in-patients with schizophrenia. Acta Psychiatr Scand 2006; 114:265-273. 
(11) Hamann J, Cohen R, Leucht S, Busch R, Kissling W. Shared decision making and longterm outcome in schizophrenia treatment. J Clin Psychiatry 2007; 68:992-997.

(12) Priebe S, McCabe R, Bullekamp J, Hansson L, Lauber C, Martinez-Leal R, Rossler W, Salize H, Svensson B, Torres-Gonzalez F, Van den Brink R, Wiersma D, Wright DJ. Structured patient-clinician communication and 1-year outcome in community mental healthcare. Cluster randomised controlled trial. Br J Psychiatry 2007 191:420-426.

(13) Hamann J, Mendel R, Meier A, Asani F, Pausch E, Leucht S, Kissling W. "How to speak to your psychiatrist": shared decision-making training for inpatients with schizophrenia. Psychiatr Serv 2011 62:1218-1221.

(14) Woltmann EM, Wilkniss SM, Teachout A, McHugo GJ, Drake RE. Trial of an Electronic Decision Support System to facilitate shared decision making in community mental health. Psychiatr Serv 2011 62:54-60.

(15) Drake RE, Cimpean D, Torrey WC. Shared decision making in mental health: prospects for personalized medicine. Dialogues Clin Neurosci 2009 11:455-463.

(16) Berghmans RLP. Capacity and consent. Curr Opin Psychiatry 2001 14:491-499.

(17) Simón-Lorda P. La capacidad de los pacientes para tomar decisiones: una tarea todavía pendiente. Rev Asoc Esp Neuropsiq 2008 28,102:325-348.

(18) Jeste DV, Depp CA, Palmer BW. Magnitude of impairment in decisional capacity in people with schizophrenia compared to normal subjects: an overview. Schizophr Bull 2006 32:121128.

(19) Stiles PG, Poythress NG, Hall A, Falkenbach D, Williams R. Improving understanding of research consent disclosures among persons with mental illness. Psychiatr Serv 2001 52:780-785.

(20) Roberts LW, Geppert CMA, Bailey R. Ethics in psychiatric practice: essential ethics skills, informed consent, the therapeutic relationship, and confidentiality. J Psychiatr Pract 2002 8:290-305.

(21) Ganzini L, Volicer L, Nelson W, Derse A. Pitfalls in assessment of decision-making capacity. Psychosomatics 2003 44,3:237-243.

(22) Ganzini L, Volicer LA, Nelson WA, Fox E, Derse AR. Ten myths about decision-making capacity. J Am Med Dir Assoc 2004 5,4:263-267.

(23) Roth LH, Meisel A, Lidz CW. Tests of competency to consent to treatment. Am J Psychiatry 1977 134:279-284.

(24) Appelbaum PS, Roth LH. Competency to consent to research: a psychiatric overview. Arch Gen Psychiatry 1982 39:951-958.

(25) Appelbaum PS, Grisso T. Assessing patient's capacities to consent to treatment. N Eng J Med 1988 319,25:1635-1638.

(26) Alexander MP. Clinical determination of mental competence. A theory and a retrospective study. Arch Neurol 1988 45:23-26.

(27) Freedman M, Stuss DT, Gordon M. Assessment of competency: the role of neurobehavioral deficits. Ann Intern Med 1991 115:203-208.

(28) Drane JF. The many faces of competency. Hastings Cent Rep 1985 15,2:17-21.

(29) Drane JF. Las multiples caras de la competencia. En: A. Couceiro, editor Bioética para clínicos. Madrid: Triacastela, 1999; p.163-176.

(30) Demarco JP. Competence and paternalism. Bioethics 2002 16,3:231-245. 
ORIGINALES Y REVISIONES

(31) Moye J, Gurrera RJ, Karel MJ, Edelstein B, O'Connell C. Empirical advances in the assessment of the capacity to consent to medical treatment: clinical implications and research needs. Clin Psychol Rev 2006 26:1054-1077.

(32) Marson DC, Cody HA, Ingram KK, Harrell LE. Neuropsychological predictors of competency in Alzheimer's disease using a rational reasons legal standard. Arch Neurol 1995 52:955-959.

(33) Appelbaum PS. Assessment of patients' competence to consent to treatment. N Eng J Med 2007 357:1834-1840.

(34) Sturman ED. The capacity to consent to treatment and research: a review of standardized assessment tools. Clin Psychol Rev 2005 25:954-974.

(35) Dunn LB, Nowrangi MA, Palmer BW, Jeste DV, Saks ER. Assessing decisional capacity for clinical research or treatment: a review of instruments. Am J Psychiatry 2006 163:1323-1334.

(36) Kim SY, Caine ED. Utility and limits of the mini mental state examination in evaluating consent capacity in Alzheimer's disease. Psychiatr Serv 2002 53,10:1322-1324.

(37) Bean G, Nishisato S, Rector NA, Glanzy G. The assessment of competence to make a treatment decision: an empirical approach. Can J Psychiatry 1996 41:85-92.

(38) Janofsky JS, McCarthy RJ, Folstein MF. The Hopkins competency assessment test: a brief method for evaluating patient's capacity to give informed consent. Hosp Community Psychiatry 1992 43:132-136.

(39) Carney MT, Neugroschl J, Morrison RS, Marin D, Siu AL. The development and piloting of a capacity assessment tool. J Clin Ethics 2001 12:17-23.

(40) Edelstein B. Hopemont capacity assessment interview manual and scoring guide. Morgantown, WV: West Virginia University, 1999.

(41) Wong JG, Clare ICH, Holland AJ, Watson PC, Gunn M. The capacity of people with a 'mental disability' to make a health care decision. Psychol Med 2000 30:295-306.

(42) Etchells E, Darzins P, Silberfeld M, Singer PA, McKenny J, Naglie G, Katz M, Guyatt GH, Molloy W, Strang D. Assessment of patient capacity to consent to treatment. J Gen Intern Med 1999 14:27-34.

(43) Grisso T, Appelbaum A. MacArthur competency assessment tool for treatment (MacCAT-T). Sarasota, FL:Professional Resource Press, 1998.

(44) Hernando P, Lechuga X, Solé P, Diestre G, Mariné A, Rodríguez A, Marquina D, Colomer O. Validación, adaptación y traducción al castellano del MacCAT-T: herramienta para evaluar la capacidad en la toma de decisiones sanitarias. Rev Calidad Asistencial 2012 27,2:85-91.

(45) Owen G, Szmukler G, Richardson G, David AS, Hayward P, Rucker J, Harding D, Hotopf M. Mental capacity and psychiatric in-patients: Implication for the new mental health law in England and Wales. Br J Psychiatry 2009 195,3:257-263.

(46) Richardson G. Balancing autonomy and risk: a failure of nerve in England and Wales? Int J Law Psychiatry 2007 30.1:71-80.

(47) Owen G, Richardson G, David AS, Szmukler G, Hayward P, Hotopf M. Mental capacity to make decisions on treatment in people admitted to psychiatric hospitals: cross-sectional study. BMJ 337;448, 2008 doi:10.1136/bmj.39580.546597.BE.

(48) Cairns R, Maddock C, Buchanan A, David AS, Hayward P, Richardson G, Szmukler G, Hotopf M. Prevalence and predictors of mental incapacity in psychiatric in-patients. Br J Psychiatry 2005 187:379-385. 
(49) Raymont V, Bingley W, Buchanan A, David AS, Hayward P, Wessely S, Hotopf M. The prevalence of mental incapacity in medical inpatients and associated risk factors: cross-sectional study. Lancet 2004 364:1421-1427.

(50) Okai D, Owens G, McGuire H, Singh S, Churchill R, Hotopf M. Mental capacity in psychiatric patients. Br J Psychiatry 2007 191:291-297.

(51) Fassassi S, Bianchi Y, Stiefel F, Waeber G. Assessment of the capacity to consent to treatment in patients admitted to acute medical wards. BMC Med Ethics 10:15, 2009 doi: 10.1186/1.472-6939-10-15.

(52) Szmukler G. "Personality disorder" and capacity to make treatment decisions. J Med Ethics 200935:647-650.

(53) Szmukler G, Holloway F. Reform of the Mental Health Act: health or safety? Br J Psychiatry 2000 177:196-200.

(54) Shah A, Pennington M, Heginbotham C, Donaldson D. Deprivation of liberty safeguards in England: implementation costs. Br J Psychiatry 2011 199:232-238.

(55) Expert Committee. Review of the Mental Health Act 1983. London: Department of Health, 1999. 